\title{
In-vitro assessment of hemocompatibility and enzyme compatibility of hydroxyapatite nanoparticles
}

\author{
Gamagedara TP* \\ Dept. of Basic Science, Faculty of Allied Health Sciences, University of Peradeniya, Augusta Hill, Peradeniya, Sri Lanka
}

Global demand for bone grafts is always very high due to the need of repairing deteriorated or damaged bones as a result of various traumas, natural aging, accidents and war situations. Synthetic hydroxyapatite (HA), is emerging as the most promising bioceramic material for orthopedic applications due to its similarity to the inorganic matrix of living bone and teeth [1]. Calcium phosphate biomaterials, mainly $\mathrm{HA}$, find many clinical applications in the repair of bone defects, bone augmentation and coating for metal implants [2].

Nanotechnology is the conception and control of matter at dimensions of roughly $1-100 \mathrm{~nm}$. Nanotechnology contributes to investigate unique and relatively new phenomena and providing novel applications. Engineered nanoparticles were considered as an important class of new materials with several properties which attracted clinicians to work on them in the last decade. Nanocrystalline calcium phosphate apatites are the key elements in bio-mineralization and biomaterials field [3]. Nanoscaled HA has displayed unique functions differing substantially from those of bulk or microscale $\mathrm{HA}$, for example, increased fracture toughness and hardness of HA ceramic, improved osseointegrative property on nanostructured HA ceramic, enhanced adsorption capacity to drug/gene/protein of apatite nano carrier and high solubility in acidic $\mathrm{pH}$ environment of cellular endolysosomes [4]. Nanostructured HA possesses higher specific surface areas that enhance the adhesion of cells, proteins, and drugs. Parameters such as material, size, surface chemistry and shape have been engineered to tailor various attributes of nanoparticles including their ability to circulate in blood, target specific tissues, internalize into target cells and release drugs [5]. Size is an important parameter in the design of long-circulating nanoparticles. It is generally accepted that particles with a size of less than $10 \mathrm{~nm}$ are easily removed from the body by renal excretion. Shape is now recognized as a crucial parameter in determining the behavior of nano/microparticles in various processes including blood circulation, targeting, cellular uptake and intracellular trafficking [5]. Hence miniaturization of size and tuning of the morphology are an important factor for current biomedical research [6]. Ability of amphiphilic compounds to self-assemble into welldefined structures can be applied for the synthesis of nanomaterials with nanometer dimensions. It was found that the surfactant templates played an important role in regulating the morphology of nanoparticles [7]. It is a fact that the reproducible preparation of stable particles with controlled/ pre-chosen size is a very difficult task using popular colloidal-chemical approach [8]. If simpler methods are investigated to synthesize homogeneous hydroxyapatite nanoparticles, industrial production for biomedical applications can be encouraged.

The adverse biological effect of nanoparticles is an unavoidable scientific problem because of their small size and high surface activity. There are some studies revealing that the potential of nanoparticles to alter normal physiology by interacting with biomolecules in living cells thereby causing adverse effects at the cellular, subcellular, and protein levels [9]. Entering of NPs into the vascular system intentionally and interacting with blood cannot be neglected. The wear nanoparticles would be prone to binding with proteins to form protein-particle complexes, to interacting with visible components in the blood including erythrocytes, leukocytes, and platelets, and to being phagocytosed by macrophages or fibroblasts to deposit in the local tissue, leading to the formation of fibrous local pseudocapsules. These particles would also be translocated and disseminated into the main organs such as the lung, liver and spleen via blood circulation [9].

NPs, when entered into the body, will interact initially with proteins present in biological fluids, such as blood. Protein adsorption onto NPs in biological medium has been taken into consideration as an important factor for the assessment of biological responses to NPs. Binding to plasma components and forming a nanoparticle-protein corona potentially determine the fate of NPs in the systemic circulation and influence their biological activity. In human plasma, a typical protein corona formed on NPs consists of proteins such as serum albumin, immunoglobulins, fibrinogen, and apolipoproteins, etc [9].

With the advent and rapid development of nanoscience and nanotechnology, every synthetic event occurs at the nanoscale. Especially the engineered nanoparticles (NPs) are massively produced and widely used in the fields of electronics, environment, agriculture, pharmacy, and medicine, etc [10]. In the medical field, nanotechnologies are currently being developed for diagnosing and treating diseases. However, most nanoparticles do not reach their intended target and are sequestered by the liver and spleen (if $>6 \mathrm{~nm}$ ) or eliminated though the kidney (if $<6 \mathrm{~nm}$ ) after administration into the body [11]. There is a study shows that intravenously administrated radiolabelled HA NPs was distributed everywhere in the body with blood circulation, but mainly accumulated in the lung, liver and spleen. The special biochemical parameters in serum partially reflect the function of these organs, such as alanine aminotransferase (ALT) and aspartate aminotransferase (AST) for liver function, blood urea nitrogen (BUN) for kidney function and alkaline phosphatase (ALP) for liver function or bone formation-related diseases, and so on [12].

Biological assessment requires candidate materials to be noncytotoxic and to be bio functional, able to elicit a beneficial hostresponse in a specific application [13]. There are several in vitro and

${ }^{*}$ Correspondence to: Gamagedara TP, Dept. of Basic Science, Faculty of Allied Health Sciences, University of Peradeniya, Augusta Hill, Peradeniya, Sri Lanka, E-mail: tpiumnil@pdn.ac.lk

Received: September 27, 2018; Accepted: October 05, 2018; Published: October 08,2018 
in vivo studies which have been performed to identify whether there is a toxic effect of HA NPs when it is used as a bone replacement or therapeutic material. When HA NPs are used for bone replacement, biocompatibility with blood components (haemocompatibility), enzymes and bone cells have to be ensured.

\section{References}

1. Vallet-Regí M (2001) Ceramics for medical applications. J Chem Soc Dalt Trans 2: 97-108.

2. Gamagedara TP, Rathnayake UM, Rajapakse RMG (2018) Facile synthesis of hydroxyapatite nanoparticles by a polymer-assisted method? morphology, mechanical properties and formation mechanism. $J$ Clin Invest 1: 1-5.

3. Bayani M, Torabi S, Shahnaz A, Pourali M (2017) Main properties of nanocrystalline hydroxyapatite as a bone graft material in treatment of periodontal defects. A review of literature. Biotechnol Biotechnol. Equip 31: 215-220.

4. Han Y, Li S, Cao X, Yuan L, Wang Y, et al. (2014) Different inhibitory effect and mechanism of hydroxyapatite nanoparticles on normal cells and cancer cells in vitro and in vivo. Sci Rep 4:1-8. [Crossref]

5. Yoo JW, Chambers E, Mitragotri S (2010) Factors that control the circulation time of nanoparticles in blood: challenges, solutions and future prospects. Curr Pharm Des 16: 2298-2307. [Crossref]
6. Manoj M, Subbiah R, Mangalaraj D, Ponpandian N, Viswanathan C, et al. (2015) Influence of Growth Parameters on the Formation of Hydroxyapatite (HAp) Nanostructures and Their Cell Viability Studies. Nanobiomedicine (Rij) 2: 2. [Crossref]

7. Holmberg K (2004) Surfactant-templated nanomaterials synthesis. J Colloid Interface Sci 274: 355-364.

8. Z LW, Tapan TP, Sau K, Anjali Pal, Jana NR (2001) Size controlled synthesis of gold nanoparticles using photochemically prepared seed particles. J Nanoparticle Res 3 : $257-261$.

9. Adeyemi OS, Adewumi I (2014) Biochemical Evaluation of Silver Nanoparticles in Wistar Rats. Int Sch Res Notices 2014: 196091. [Crossref]

10. Ilinskaya AN, Dobrovolskaia MA (2013) Nanoparticles and the blood coagulation system. Part II: Safety concerns. Nanomedicine 8: 969-981. [Crossref]

11. Zhang YN, Poon W, Tavares AJ, McGilvray ID, Chan WCW (2016) Nanoparticle-liver interactions: Cellular uptake and hepatobiliary elimination J Control Release 240: 332348. [Crossref]

12. Albanese A, Tang PS, Chan WC (2012) The effect of nanoparticle size, shape, and surface chemistry on biological systems. Annu Rev Biomed Eng 14: 1-16. [Crossref]

13. Herath HMTU, Di Silvio L, Evans JRG (2005) Porous hydroxyapatite ceramics for tissue engineering. J Appl Biomater Biomech 3: 192-198.

Copyright: $(2018$ Gamagedara TP. This is an open-access article distributed under the terms of the Creative Commons Attribution License, which permits unrestricted use, distribution, and reproduction in any medium, provided the original author and source are credited. 